\title{
Perinatal Assessment of Cerebral Flow Velocity Wave Forms in the Human Fetus and Neonate
}

\author{
GREG CONNORS,' CORA HUNSE, ROBERT GAGNON, BRYAN RICHARDSON, VICTOR HAN, \\ AND HERSCH ROSENBERG \\ Lawson Research Institute, St. Joseph's Health Centre, Departments of Obstetrics and Gynecology /G.C., C.H., \\ R.G., B.R.] and Pediatrics [V.H., H.R.], University of Western Ontario, London, Ontario, Canada
}

\begin{abstract}
The temporal relationship between changes in cerebral Doppler flow velocity wave forms, ductal patency, blood gases, and blood pressure during the transition from intrauterine to newborn life was assessed longitudinally in 16 healthy term fetuses and newborns. Doppler flow velocity wave forms were obtained from fetal cerebral arteries (anterior cerebral, internal carotid, and basilar) before birth, within $8 \mathrm{~h}$ after birth, and again at 24 and 48 $h$ after birth. The resistance index was used as a measure of vascular resistance. The resistance index of the cerebral arteries studied increased significantly between the antenatal and 8-h study periods. This was followed by a significant decrease below fetal levels by the $24-\mathrm{h}$ study period, with little change thereafter. We conclude that in the newborn human, as in the newborn lamb, the transition from fetal to immediate newborn life is associated with an increase in cerebral vascular resistance and thus a decrease in cerebral blood flow in response to the increase in arterial oxygenation. The subsequent decrease in the cerebral resistance index between 8 and $24 \mathrm{~h}$ of life cannot be explained by a loss of ductal shunting nor by associated changes in newborn blood gases or blood pressure, but may rather reflect a remodeling of the circulation due to impedance matching. (Pediatr Res 31: 649-652, 1992)
\end{abstract}

\section{Abbreviations}

DFVW, Doppler flow velocity wave forms $R I$, resistance index

PDA, patent ductus arteriosus

MPA, main pulmonary artery

AAO, ascending aorta

MV, mean velocity

The transition from intrauterine to newborn life is associated with profound changes in blood flow patterns due to removal of the placental circulation, the inflation of the lungs, and subsequent changes in the blood gas composition. In sheep, the transition from prenatal to immediate postnatal life is associated with a progressive decrease in cerebral blood flow over the first $24 \mathrm{~h}$ of life, which correlates inversely with the postnatal rise in arterial oxygen content (1). This decrease in blood flow in turn reflects an increase in cerebral vascular resistance, inasmuch as mean arterial pressure remains little changed.

In the human, Doppler resistance indices as measured from

Received September 16, 1991; accepted January 14, 1992.

Correspondence: Greg Connors, M.D., Department of Obstetrics and Gynecology, Heritage Medical Research Building, University of Calgary, 3330 University Drive N.W. Calgary, Alberta T2N 4N

Supported by grants from the Canadian Medical Research Council.

'Canadian Medical Research Council Fellow.
DFVW are felt to reflect downstream vascular resistance (2) and, thus, provide a noninvasive means of assessing the fetal/neonatal circulation. In the human neonate, the cerebral RI, measured from the DFVW, has been shown to decrease over the first $24 \mathrm{~h}$ of life, with little change thereafter $(3,4)$. More recently, it has been reported in healthy term pregnancies that the cerebral DFVW indices are increased during the first $24 \mathrm{~h}$ of life compared with fetal values and subsequently decreased during the second day of life $(5,6)$. In these reports $(3,5)$, the increased cerebral RI during the first $24 \mathrm{~h}$ of life, when compared with fetal and later neonatal values, was felt to reflect "diastolic run off" associated with a PDA rather than a response of vascular resistance to changing oxygenation. Although the association of low diastolic velocities and increased Doppler indices has been reported for clinically and hemodynamically significant PDA (7), the effect of clinically "insignificant" PDA on Doppler indices is not known. The lower neonatal (5) and adult (8) cerebral DFVW resistance indices compared with fetal values, when measured cerebral vascular resistance must in fact be higher, also remains to be explained.

The purpose of this study was, therefore, to assess the temporal relationship between changes in cerebral DFVW and ductal patency during the transition from intrauterine to newborn life. Blood gases and arterial blood pressure were also monitored during the neonatal period as additional factors possibly affecting the perinatal change in cerebral DFVW indices.

\section{MATERIALS AND METHODS}

Sixteen healthy pregnant women undergoing elective induction of labor or cesarean section were studied. Four women were delivered by cesarean section under epidural anesthesia (all elective repeat), and the remaining 12 women were induced electively at term or for postdates. Fetal outcome confirmed the gestational age and well being of the fetuses and newborns studied (Table 1). This protocol was approved by the University of Western Ontario Bioethics Committee on human research, and informed consent was obtained from all participants.

Studies were initiated either the evening or morning before

Table 1. Clinical data on study patients*

$\begin{array}{lc}\text { Mean gestation at birth (wk) } & 41.0 \pm 0.1 \\ \text { Birth route } & 12 \\ \quad \text { Vaginal } & 4 \\ \quad \text { Cesarean section } & 3523 \pm 26 \\ \text { Birth weight }(\mathrm{g}) & \\ \text { Umbilical artery } & 7.25 \pm 0.006 \\ \mathrm{pH} & 3.3 \pm 0.2(25.0 \pm 1.3) \\ \mathrm{PO}_{2}[\mathrm{kPa}(\mathrm{mm} \mathrm{Hg})] & 6.2 \pm 0.2(46.8 \pm 1.4) \\ \mathrm{PCO}_{2}[\mathrm{kPa}(\mathrm{mm} \mathrm{Hg})] & -5.0 \pm 0.3 \\ \mathrm{BE} & \end{array}$

* Data presented as mean $\pm \operatorname{SEM}(n=16)$. BE, base excess. 
elective induction of labor or cesarean section. Each patient study consisted of four study periods; antenatal, and within 8 , 24 , and $48 \mathrm{~h}$ postnatal. The antenatal study measurements were made within $24 \mathrm{~h}$ of birth, but before the onset of labor, whereas the $8 \mathrm{~h}$ study measurements were made within $8 \mathrm{~h}$ of birth after the newborn assessment and the initiation of parental bonding. Measurements were then repeated at approximately 24 and $48 \mathrm{~h}$ after birth for the 24- and 48-h study periods.

The antenatal study period consisted of DFVW measurements obtained from the fetal cerebral arteries; anterior cerebral, internal carotid, and basilar. During the 8 -h study period, DFVW were again obtained from the cerebral arteries, and patency of the ductus arteriosus was determined using two-dimensional, pulsed Doppler, and color flow mapping ultrasound as well as DFVW measurements from the proximal MPA and AAO. Ductal patency was diagnosed when flow could be detected at the pulmonary end of the ductus arteriosus with either pulsed Doppler or color flow ultrasound. Newborn blood pressure, heart rate, and transcutaneous $\mathrm{PO}_{2}$ and $\mathrm{PCO}_{2}$ were additionally monitored. These newborn measurements were then repeated for the 24- and 48-h study periods.

In addition to identifying ductal patency, we attempted to quantitate ductal shunting with the ratio of AAO MV to MPA MV, as measured from their respective DFVW. The MV was calculated as the time averaged mean of the maximum velocity for each DFVW. Because left to right ductal shunting will be associated with increased left ventricular output compared with right ventricular output, the ratio of left to right ventricular output should provide an assessment of ductal shunting. Ventricular output $(\mathrm{CO})$ can be calculated from the following equation: $\mathrm{CO}=\mathrm{MV} \times 60 \times$ vessel cross-sectional area. Because the area of the aortic root does not change over the first $48 \mathrm{~h}$ of life (9), and if we assume this is also true for the MPA, the ratio of left to right ventricular output may be simplified to the ratio of AAO MV to MPA MV. Therefore, the greater the degree of ductal shunting, the more this ratio will vary from unity.

DFVW measurements consisted of up to three observations of three to five consecutive DFVW, with a mean value then obtained for each of the vessels studied during each study period. The RI, as measured from the DFVW, was used as an index of downstream vascular resistance $[R I=(S-D) / S$ where $S=$ peak systolic and $\mathrm{D}=$ end diastolic] (10). DFVW measurements were made using real-time, pulsed Doppler, and color flow mapping ultrasound with $3.0-\mathrm{MHz}$ (fetal studies) and $5.0-\mathrm{MHz}$ (newborn studies) scan heads (Ultrasmark 9; Advanced Technologies Laboratories, Bothell, WA). The fetal cerebral vessels were insonated in the axial plane at the level of the cerebral peduncles. The internal carotid artery was insonated just proximal to its bifuration into the anterior and middle cerebral arteries, with the anterior cerebral artery being insonated immediately distal to this bifurcation. The basilar artery was insonated between the cerebral peduncles just proximal to its bifurcation into the posterior cerebral arteries. During the newborn study periods, the cerebral vessels were again insonated in the axial plane at the level of the cerebral peduncles, thus allowing these vessels to be insonated at the same site as for the fetal study. This was accomplished by using a transcranial technique and placing the transducer approximately in the temporal region between the ear and eye. The ductus arteriosus, MPA, and AA were imaged using standard echocardiographic windows. DFVW were obtained from the MPA and AAO by placing the sample volume just distal to the valve and maintaining an angle of insonation less than $10^{\circ}$. This information was not recorded for all patients studied. As the study progressed, the need to assess ductal shunting became obvious. The incidence of PDA was recorded in all but the first two patients. The "quantitation" of ductal shunting was recorded for the last seven patients studied.

Fetal and newborn heart rates were calculated from the cardiac cycle length of the respective DFVW. Measurements were averaged to provide a mean value for each patient during each of the study periods. Newborn blood pressure was automatically recorded from the left upper arm with a Dinamap (model 845XT; Crititen, Inc., Tampa, FL) every 5 min during the newborn study periods. Measurements were averaged to provide a mean value for each patient during each of the three neonatal study periods. The $\mathrm{PO}_{2}$ and $\mathrm{PCO}_{2}$ for the newborn study periods were also recorded every $5 \mathrm{~min}$ using a transcutaneous $\mathrm{PO}_{2}, \mathrm{PCO}_{2}$ monitor (Kontron Scientific Ltd., Calgary, Alberta, Canada) with the transcutaneous electrodes placed on the right chest just below the nipple. Once again, measurements were averaged, providing a mean value for each patient during the DFVW measurements.

Data analysis. Results are presented as grouped means and SEM for the RI, MV, blood pressure, heart rate, $\mathrm{PO}_{2}$, and $\mathrm{PCO}_{2}$ values for each study period. Statistical significance between study periods was determined using a nonpaired $t$ test with a Bonferroni correction for multiple comparisons.

\section{RESULTS}

The mean gestational age at the initiation of the study was $41.0 \pm 0.07 \mathrm{wk}$. The mean, SEM, and range of observation times for the four study periods are as follows: antenatal, $20.0 \pm 0.5$ (2.3-36.6) $\mathrm{h}$ before birth, but before the onset of labor; $8 \mathrm{~h}$, $4.6 \pm 0.2(1.4-9.6) \mathrm{h}$ after birth; and 24 and $48 \mathrm{~h}, 23.9 \pm 0.3$ $(17.2-29.0)$ and $47.5 \pm 0.3(39.7-55.4) \mathrm{h}$ after birth, respectively.

The RI of the cerebral vessels studied (anterior cerebral, internal carotid, and basilar arteries) increased significantly from the antenatal period to $8 \mathrm{~h}$ of newborn life (Fig. 1, Table 2). Between the 8 - and 24-h study periods, there was a significant decrease in

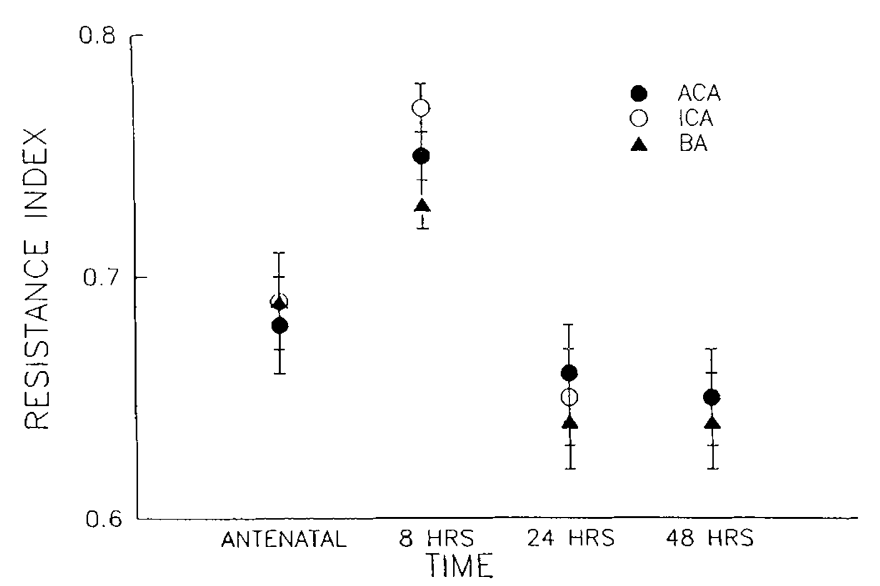

Fig. 1. The RI data (mean $\pm \mathrm{SEM}$ ) for the cerebral arteries studied ( $A C A$, anterior cerebral; $I C A$, internal carotid; and $B A$, basilar) for the antenatal and three newborn study periods $(n=16)$.

Table 2. Resistance index and heart rate data*

\begin{tabular}{llccc}
\hline & Antenatal & $8 \mathrm{~h}$ & $24 \mathrm{~h}$ & $48 \mathrm{~h}$ \\
\hline RI & & & & \\
ACA & $0.68 \pm 0.02 \dagger$ & $0.75 \pm 0.01$ & $0.66 \pm 0.02 \ddagger$ & $0.65 \pm 0.02 \ddagger$ \\
ICA & $0.69 \pm 0.02 \ddagger$ & $0.77 \pm 0.01$ & $0.65 \pm 0.02 \ddagger$ & $0.65 \pm 0.02 \ddagger$ \\
BA & $0.69 \pm 0.02$ & $0.73 \pm 0.01$ & $0.64 \pm 0.02 \ddagger$ & $0.64 \pm 0.02 \ddagger$
\end{tabular}

Heart rate

$(\mathrm{bpm})$

$\begin{array}{lllll}\text { ACA } & 130 \pm 3 & 123 \pm 4 & 121 \pm 2 & 119 \pm 3 \\ \text { ICA } & 136 \pm 2 & 121 \pm 4 \dagger & 122 \pm 3 \dagger & 118 \pm 4 \ddagger \\ \text { BA } & 132 \pm 2 & 123 \pm 4 & 121 \pm 3 & 118 \pm 4 \ddagger\end{array}$

* Data presented as means \pm SEM for the cerebral arteries studied during the four study periods. ACA, anterior cerebral; ICA, internal cerebral; BA, basilar. RI data significance was determined by comparison with the 8 -h study group. Heart rate data significance was determined by comparison with the antenatal study group $(n=16)$.

$\dagger p<0.05$.

$\ddagger p<0.01$. 
the cerebral RI for the vessels studied, with little change observed between 24 and $48 \mathrm{~h}$ postnatally. Although the newborn cerebral $\mathrm{RI}$ at 24 and $48 \mathrm{~h}$ postnatal are lower than the fetal values observed during the antenatal period, they were not statistically different. Similar changes were observed in all three of the cerebral vessels studied.

The mean heart rate for each cerebral vessel studied for each of the study periods is shown in Table 2 . There was a decrease in heart rate from the antenatal to the 48 -h study period; this trend was significant for the internal carotid and basilar arteries. Neonatal blood pressure and transcutaneous $\mathrm{PO}_{2}$ and $\mathrm{PCO}_{2}$ measurements for the three newborn study periods are shown in Table 3 . Systolic and diastolic blood pressure increased slightly over the first $48 \mathrm{~h}$ of life; this increase did not reach statistical significance. No change in pulse pressure was observed over the newborn study periods. Similarly, no significant changes in $\mathrm{PO}_{2}$ or $\mathrm{PCO}_{2}$ were observed over the newborn study periods.

To assess the effect of ductus arteriosus shunting on the cerebral RI, the MV of the AAO and MPA were measured and their ratio determined, along with the incidence of ductal patency during each of the newborn study periods (Table 4). As expected, the incidence of PDA decreased from 8 to $48 \mathrm{~h}$ of life. Although the ratio of AAO to MPA MV did decrease slightly over the first $48 \mathrm{~h}$, this trend did not reach statistical significance. In addition, two newborn patients were found to have a closed ductus arteriosus during all three newborn study periods. The mean internal carotid artery RI of both of these patients demonstrated a similar trend and degree of change when compared with the rest of the patients over the four study periods (antenatal, 0.67 versus $0.69 \pm 0.02 ; 8 \mathrm{~h}, 0.73$ versus $0.77 \pm 0.01 ; 24 \mathrm{~h}, 0.64$ versus $0.65 \pm 0.22$; and $48 \mathrm{~h}, 0.61$ versus $0.65 \pm 0.02$ ). The RI for the anterior cerebral and basilar arteries showed the same trend over the four study periods.

\section{DISCUSSION}

In this study, we observed that the cerebral RI increased significantly between the antenatal and 8-h postnatal study periods. This was followed by a significant decrease in the cerebral RI, which fell below the fetal values at $24 \mathrm{~h}$, with no further change observed at $48 \mathrm{~h}$ postnatally. Similar changes were noted in all three cerebral vessels studied. Our findings are consistent with those reported by Lowery et al. (5) for the internal carotid artery and Meerman et al. (6) for the middle cerebral artery over the same perinatal period. Wright et al. (3) have also reported on the significant decrease in the RI of the internal carotid and anterior cerebral arteries between 8 and $24 \mathrm{~h}$ of newborn life, and the newborn study of Shuto et al. (4) demonstrated a similar decrease in the internal carotid RI over the first $12 \mathrm{~h}$ of newborn life, with no further change over the first 2 wk of life.

Although all of these studies report similar changes in cerebral DFVW RI over the perinatal or newborn period, the mechanism underlying these changes remains to be determined. It has been proposed that the increase in cerebral DFVW RI over the first few hours of newborn life and the subsequent decrease thereafter reflect vascular changes within the ductus arteriosus and the effect of "diastolic run off" on downstream blood flow rather than a direct effect of changing cerebral vascular resistance. With left to right shunting through the ductus arteriosus at birth, the DFVW of the cerebral vessels would be altered by a lowering of the end diastolic velocity due to diastolic run off without significantly effecting the peak systolic velocity, resulting in an increase in the DFVW RI. With the subsequent closure of the ductus arteriosus over the early neonatal period, these SC DFVW indices would presumably return to prenatal levels. Indeed, Perlman et al. (7) have reported that closure of clinically significant PDA are associated with a reduction in the anterior cerebral artery DFVW RI.

In the present study, none of the infants experienced any complications during the newborn period. Those infants in whom ductal patency was determined by ultrasound examination (all of whom closed by $48 \mathrm{~h}$ ) were thus felt to have clinically "insignificant" PDA. This is consistent with data reported by Gentile et al. (11), where $72 \%$ of newborns in whom PDA was determined by pulsed Doppler ultrasound displayed no clinical signs of symptoms of ductal patency. To determine what role these clinically insignificant PDA played in the changes that we observed in the cerebral RI, we endeavored to evaluate the temporal change in ductal patency and/or hemodynamic significance. First, as shown in Table 3, there was no significant change in the diastolic or pulse pressures over the newborn study periods. Second, although we did observe a decreasing trend in the AAO/ MPA MV (Table 4), this was not statistically significant. This trend is consistent with closure of the clinically insignificant PDA observed in this study. Additionally, two newborn study infants were found to have closed ductus arteriosus during all three newborn study periods. These newborns still demonstrated the same changes in the cerebral RI as those newborns in which ductal patency was observed. This data thus suggests that the hemodynamics of the ductus arteriosus cannot account for the perinatal change in the cerebral RI normally observed in the healthy newborn infant.

Heart rate has also been shown to effect DFVW indices. However, the small change in heart rate reported here is unlikely to be a factor in the perinatal RI changes, inasmuch as we have shown that although there is an independent inverse relationship of heart rate on the RI it accounts for less than a 3\% change in RI over the fetal heart rate range of 120 to $160 \mathrm{bpm}$ (12). Because cerebral blood flow, and thus vascular resistance, is coupled to cerebral metabolic activity (13), a change in such activity might also contribute to the perinatal change in cerebral RI observed here. Although cerebral metabolic activity was not directly monitored, little change would be expected because all newborn studies were carried out at the same level of behavioral activity (quiet awake or quiet asleep) and experimental data in the ovine species indicates little change in cerebral oxygen consumption over the immediate perinatal period (1).

In the ovine fetus, Richardson et al. (1), using the radiolabeled microsphere technique, reported the transition from prenatal to early postnatal life to be associated with a progressive decrease in cerebral blood flow. This decrease in blood flow reflected an increase in vascular resistance, because mean arterial pressure remained little changed and showed an inverse relationship to the measured increase in arterial oxygen content. This is a welldescribed relationship for both the fetal (14) and neonatal (15) lamb and serves to maintain oxygen delivery to the brain with

Table 3. Neonatal blood pressure and transcutaneous $\mathrm{PO}_{2}$ and $\mathrm{PCO}_{2}$ data*

\begin{tabular}{lccc}
\hline & $8 \mathrm{~h}$ & $24 \mathrm{~h}$ & $48 \mathrm{~h}$ \\
\hline Blood pressure (mm Hg) & & & \\
$\quad$ Systolic & $64.2 \pm 3.3$ & $68.8 \pm 3.3$ & $74.0 \pm 2.3$ \\
$\quad$ Diastolic & $37.1 \pm 2.3$ & $44.6 \pm 2.3$ & $45.8 \pm 2.4$ \\
$\quad$ Mean & $49.7 \pm 3.0$ & $55.3 \pm 3.5$ & $58.6 \pm 3.2$ \\
Transcutaneous gases [kPa (mm Hg)] & $9.8 \pm 0.4(73.3 \pm 3.2)$ & $9.2 \pm 0.4(69.0 \pm 2.9)$ & $9.3 \pm 0.4(70.1 \pm 3.0)$ \\
$\mathrm{PO}_{2}$ & $3.8 \pm 0.2(28.8 \pm 1.5)$ & $3.9 \pm 0.2(28.9 \pm 1.2)$ & $3.9 \pm 0.2(29.5 \pm 1.2)$ \\
$\mathrm{PCO}_{2}$ &
\end{tabular}

* Data presented as means \pm SEM for the three newborn study periods $(n=16)$. 
Table 4. Mean velocity and ductal patency data*

\begin{tabular}{lccc}
\hline & $8 \mathrm{~h}$ & $24 \mathrm{~h}$ & $48 \mathrm{~h}$ \\
\hline MV $(\mathrm{cm} / \mathrm{s})$ & & & \\
AAO & $45 \pm 3$ & $40 \pm 2$ & $40 \pm 3$ \\
MPA & $38 \pm 2$ & $37 \pm 1$ & $44 \pm 3$ \\
AAO/MPA & 1.2 & 1.1 & 0.9 \\
PDA & $5 / 7(12 / 14)$ & $3 / 7(5 / 14)$ & $0 / 7(0 / 14)$ \\
\hline
\end{tabular}

* MV data is presented as means \pm SEM. The incidence of PDA is shown for the seven newborns contributing to the MV data. The overall incidence of PDA for the three newborn study periods is shown in parentheses.

changing arterial oxygenation. The arterial oxygenation of the human newborn also increases over the early newborn period, as indicated in the present study by the change in arterial $\mathrm{PO}_{2}$ from the umbilical cord measurements at birth to the transcutaneous measurements at approximately $8 \mathrm{~h}$ of newborn life. We speculate that the increase in cerebral RI of the human newborn at this time may well reflect an increase in cerebral vascular resistance in response to such blood gas changes.

Although the initial increase in the cerebral RI can be accounted for by newborn blood gas changes, the subsequent return to prenatal levels between 8 and $24 \mathrm{~h}$ of newborn life cannot, inasmuch as little change was noted in measured transcutaneous $\mathrm{PO}_{2}$ and $\mathrm{PCO}_{2}$ at this time. This reduction in the cerebral DFVW indices, may instead reflect cerebral vascular adjustment to the postnatal reduction in cerebral blood flow, rather than a change in absolute cerebral vascular resistance. The increase in oxygen content after birth will lead to constriction of the cerebral capacitance vessels (arterioles and capillaries) as noted for the ovine fetus (1). This constriction will increase downstream vascular resistance (or impedance in the dynamic state) and thus increase the DFVW indices (2) of the cerebral vessels as noted in the present study. Initially, this constriction will lead to an impedance mismatch between the cerebral feeder vessels (arteries) and the capacitance vessels, resulting in increased velocity wave reflections (16). There is evidence that cerebral arteries can change diameter and reset vascular tone to match a new flow rate over time (17). This vascular remodeling is felt to reduce wall sheer forces and rematch vascular impedance, thus establishing a new steady state. The rematching of vessel impedance will reduce distal velocity wave reflections (16), altering the shape of the DFVW, and may therefore account for the reduction in the DFVW indices between 8 and $24 \mathrm{~h}$ of newborn life. This vascular remodeling may also explain why newborn and adult cerebral DFVW RI are lower than fetal values at a time when absolute cerebral vascular resistance should be higher.

We conclude that in the newborn human, as in the newborn lamb, the transition from fetal to immediate newborn life is associated with an increase in cerebral vascular resistance, reflecting decreased cerebral blood flow in response to the perinatal increase in arterial oxygen content. Furthermore, the subsequent decrease in the cerebral RI between 8 and 24 h of life cannot be explained by a loss of ductal shunting and does not appear to be associated with changes in newborn blood gas, blood pressure, or heart rate. We speculate that the decrease in the cerebral RI at this time may instead reflect vascular adaptation to the changes in cerebral blood flow and blood gas composition and under these conditions may not reflect absolute changes in downstream vascular resistance.

Acknowledgments. The authors thank Dr. R. Natale for his interest in this work and A. Williamson for her excellent technical assistance.

\section{REFERENCES}

1. Richardson BS, Carmichael L, Homan J, Tanswell K, Webster AC 1989 Regional blood flow change in the lamb during the perinatal period. Am J Obstet Gynecol 160:919-925

2. Burns PN 1987 Doppler flow estimations in the fetal and maternal circulations: principles, techniques and some limitations. In: Maulik D, McNellis D (eds) Doppler ultrasound measurement of maternal-fetal hemodynamics. Perinatology Press Ithaca, NY, pp 43-78

3. Wright LL, Baker KR, Hollander DI, Wright JN, Nagey DA 1988 Cerebral blood flow velocity in term newborn infants: changes associated with ductal flow. J Pediatr 112:768-773

4. Shuto H, Yasuhara A, Sugimoto T, Iwase S, Kobayashi Y 1987 Longitudinal determination of cerebral blood flow velocity in neonates with the Doppler technique. Neuropediatrics 18:218-211

5. Lowery CL, Mehta R, Wan J, Samdarshi TE, Nanda NC 1990 Serial color Doppler studies of internal carotid, renal and middle cerebral arteries in the fetus and newborn. Abstracts of the 10th Annual Meeting of the Society of Perinatal Obstetricians, Houston, TX, January 23-27, 1990

6. Meerman RJ, van Bel F, van Zwieten PHT, Oepkes D, de Ouden L 1990 Fetal and neonatal cerebral blood velocity in the normal fetus and neonate: a longitudinal Doppler ultrasound study. Early Hum Dev 24:209-217

7. Periman JM, Hill A, Volpe JJ 1981 The effect of patent ductus arteriosus on flow velocity in the anterior cerebral arteries: ductal steal in the premature newborn infant. J Pediatr 99:767-771

8. Ogawa S, Handa N, Matsumoto M, Etani H, Yoneda S, Kimura K, Kamada T 1988 Carbon dioxide reactivity of the blood flow in human basilar artery estimated by the transcranial Doppler method in normal men: a comparison with that of the middle cerebral artery. Ultrasound Med Biol 14:479-483

9. Winberg P, Jansson M, Morions L, Lundell BPW 1989 Left ventricular output during postnatal circulatory adaptation in healthy infants born at full term. Arch Dis Child 64:1374-1378

10. Planiol TH, Pourcelot L 1974 Doppler effect study of the carotid circulation In: Vlieger M. White DN, McCready VR (eds) Ultrasonics in Medicine. Excerpta Medica, Amsterdam

11. Gentile R, Stevenson G, Dooley T, Franklin D, Kawabori I, Perlman A 198 Pulsed Doppler echocardiographic determination of time of ductal closure in normal newborn infants. J Pediatr 98:443-448

12. Connors G, Gillis S, Hunse C, Gagnon R, Richardson B 1991 The interaction of behavioral state, heart rate and resistance index in the human fetus. J Dev Physiol 15:331-336

13. Richardson BS, Patrick JE, Abduljabbar H 1985 Cerebral oxidative metabolism in the fetal lamb: relationship to electrocortical state. Am J Obstet Gynecol 153:426-431

14. Jones MD, Sheldon RE, Peeters LL, Makowski EL, Meschia G 1978 Regulation of cerebral blood flow in the ovine fetus. Am J Physiol 235:H162- 166

15. Jones MD, Traystman RJ, Simmons MA, Molteni RA 1981 Effects of changes in arterial $\mathrm{O}_{2}$ content on cerebral flow in the lamb. Am J Physiol 240:H209H215

16. Nichols WW, O'Rourke MF 1990 McDonald's blood flow in arteries. Lea \& Febiger, Philadelphia, pp 251-269

17. Langille L, O'Donnell F 1986 Reductions in arterial diameter produced by chronic decreases in blood flow are endothelium-dependent. Science 23:405407 